\title{
An Artificial Immune System-Inspired Multiobjective Evolutionary Algorithm with Application to the Detection of Distributed Computer Network Intrusions
}

\author{
Charles R. Haag \\ Gary B. Lamont \\ Paul D. Williams \\ Gilbert L. Peterson \\ Department of Electrical and Computer Engineering \\ Graduate School of Engineering and Management \\ Air Force Institute of Technology (AFIT) \\ 2950 Hobson Way, Bldg. 640 \\ WPAFB, OH, 45433-7765 \\ charles.haag@langley.af.mil,[gary.lamont, paul.williams, gilbert.peterson]@afit.edu
}

\begin{abstract}
Today's signature-based intrusion detection systems are reactive in nature and storage-limited. Their operation depends upon catching an instance of an intrusion or virus and encoding it into a signature that is stored in its anomaly database, providing a window of vulnerability to computer systems during this time. Further, the maximum size of an Internet Protocol-based message requires the database to be huge in order to maintain possible signature combinations. In order to tighten this response cycle within storage constraints, this paper presents an innovative Artificial Immune System-inspired Multiobjective Evolutionary Algorithm. This distributed intrusion detection system (IDS) is intended to measure the vector of tradeoff solutions among detectors with regard to two independent objectives: best classification fitness and optimal hypervolume size. Our antibody detectors promiscuously monitor network traffic for exact and variant abnormal system events based on only the detector's own data structure and the application domain truth set, responding heuristically. Applied to the MIT-DARPA 1999 insider intrusion detection data set, our software engineered algorithm correctly classifies normal and abnormal events at a high level which is directly attributed to a detector affinity threshold.
\end{abstract}

\section{Categories and Subject Descriptors}

D.1.5 [Programming Techniques]: Object-oriented

Programming.

D.2.11 [Software Engineering]: Software Architectures Patterns.

I.5.4 [Pattern Recognition]: Applications - text processing. I.2.8 [Artificial Intelligence]: Problem Solving, Control Methods, and Search - Heuristic methods.

I.6.8 [Simulation and Modeling]: Types of Simulation Distributed.

Copyright 2007 Association for Computing Machinery. ACM acknowledges that this contribution was authored or co-authored by an employee, contractor or affiliate of the U.S. Government. As such, the Government retains a nonexclusive, royalty-free right to publish or reproduce this article, or to allow others to do so, for Government purposes only.

GECCO'07, July 7-11, 2007, London, England, United Kingdom. Copyright 2007 ACM 978-1-59593-698-1/07/0007...\$5.00.

\section{General Terms}

Algorithms, Measurement, Performance, Design, Experimentation, Security, Theory.

\section{Keywords}

Intrusion detection, computer networks, computer security, evolutionary computation, artificial immune system, multiobjective, distributed computing.

\section{PROBLEM MOTIVATION}

Signature-based intrusion detection systems (IDS) detect attacks by discovering exact matches between incoming data and a database of known attack string signatures. Their reactive nature and storage-limited database allows for only a subset of the $256^{65535}$ harmful signature combinations to be catalogued for future detection, after an attack. These constraints define our algorithm's application domain as the intrusion detection (ID) problem domain. Compounding these constraints, the trend of exact and variant nefarious signatures since 2001, through at least mid-2004, indicate exponential growth [1]. Thus, a more efficient IDS approach is required.

The IDS method of signature string evaluation is analogous to the Boolean Satisfiability Problem (SAT): the enumeration (or exhaustion) of a search space of $n$ variables against a function to determine which variables return true from that function [2]. The SAT defines our formal problem classification to be Nondeterministic Polynomial time (NP)-Hard, preventing it from being solved in polynomial time [3]. Inability to solve in polynomial time leads to the inability to solve ID deterministically as required in real-time IDS operation.. Because signature-based IDSs are deterministic, they become infeasible and we must consider a stochastic approximation solution, such as an evolutionary algorithm (EA) that possesses the unique ability to generate solutions in polynomial time.

In particular, we chose to integrate a multiobjective EA (MOEA) with an artificial immune system resulting in an innovative software engineered IDS [4]. In presenting this development, the project goals are defined in Section 2, the IDS design is discussed in Section 3, with experimental results and analysis presented in Section 4. 


\section{HYPOTHESIS}

One evolutionary algorithm (EA) of choice is the artificial immune system (AIS) because the IDS architecture is similar to the human biological immune system (BIS): a parallel and distributed adaptive system [5] for detecting and destroying antigens. In addition, we pursue a multiobjective approach because the consolidation of information into a single aggregated objective tends to lose data granularity and offer the decisionmaker only one solution vice a set of trade-off solutions. The solution approach develops a new proof-of-concept algorithm that advances the existing work of two AIS-motivated algorithms: Edge, Lamont and Raines' retrovirus algorithm (REALGO) [6] for its ability to escape local optima, with regard to string matching, and Coello and Cortés' multiobjective immune system algorithm (MISA) [5] for its employment of an AIS in a multiobjective EA context.

Our hypothesis is that the integration of REALGO and MISA, applied to an ID data set, validates an AIS-inspired multiobjective evolutionary algorithm (MOEA). This MOEA provides a set of tradeoff solutions with regard to the measurement of two independent objectives seeking a global minimum: highest network traffic classification fitness and optimal detector hypervolume. This hypothesis is composed of four measurable objectives:

1. Validate the migration of $C$ language-based REALGO and MISA into their Java equivalents;

2. Attain the highest correct classification rate possible. A heuristic-based positive value is assessed for any outcome, increasing the fitness score of the first objective of highest classification fitness. The higher a detector's effectiveness, the lower this objective's sum score is;

3. Identify a known optimal detector hypervolume. Research shows detector effectiveness is impacted by hypervolume of a particular size $[7,8]$. We seek detectors that do not stray from the pre-determined negative selection affinity threshold; hence, the lowest deviation score for our second objective;

4. Validate AIS cooperative communication within a distributed environment. As AIS detectors are rewarded for correct classification and detection, the AIS broadcasts its fittest detectors to listening AISs, for possible inclusion into their population. Validation is observation of this operation.

Upon termination of our algorithm, two vectors compose each objective's score for the set of surviving detectors in the population, which each detector is a solution point. By definition, multiobjective algorithms produce multiple solutions which may not be optimal for each objective [10]. By adjusting one solution for greater optimality, we risk decreasing the desired value of one or more other solutions. Thus, we desire a set or subset(s) of nondominated solutions through Pareto Optimality $\left(P^{*}\right)$. A solution point is considered Pareto-optimal (in a global-minimum context) if each of its objective's values is less-than-or-equal to all solution point objective values and it has at least one value, in any objective, smaller than any other solution point's value for that objective [9]. This set of Pareto-optimal points represents the Pareto Front $\left(P F^{*}\right)$ of fittest (non-dominated) detectors that is provided to the decision-maker, giving that person more options in selecting solution points for future ID domain employment based on the classification-to-hypervolume tradeoff depicted by the true $P F^{*}$.

\section{HIGH AND LOW LEVEL DESIGN}

Our IDS algorithm is the methodical integration of the foundational AIS framework, a MOEA and a modification of that EAs standard operators by REALGO and MISA [4]. The design approach employs good software engineering practice. Timmis and De Castro define a standardized AIS framework where the engineered solution is application domain-specific (see Figure 1) [10]. This framework can be thought of as a layered approach. The basis for an AIS begins with the pre-defined application (problem) domain, which governs the method of representation. Once chromosome data structure representation (e.g., bit string, real-valued vector, length, etc.) is decided, one or more affinity measures are used to quantify interactions of the system's elements; e.g., Hamming distance measurements applies to bit string representation while Euclidian distance is applied to realvalued vectors. The top layer, immune algorithms, encompasses those functions that govern the behavior (dynamics) of the system; e.g., method of mutation, selection, evaluation, etc. Addressing these layers leads to a engineered domain-specific solution.

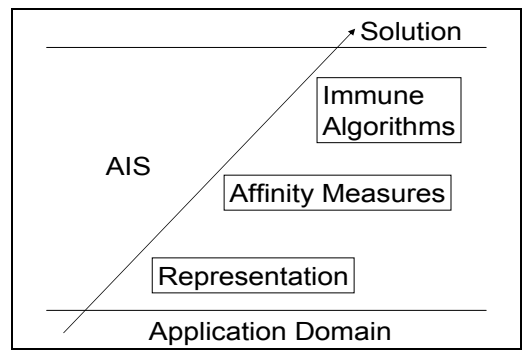

Figure 1. Timmis’ AIS framework [10]

\subsection{Application Data Domain}

The application domain is composed of week one and two of the MIT-DARPA (LL) 1999 insider intrusion detection data set [11] because it currently constitutes the largest publicly available benchmark of network traffic [12]. The first week consists of normal (self)-only traffic, facilitating negative selection. The second week totals 7.2 million packets composed of $99.25 \%$ self and $0.75 \%$ labeled attacks (non-self), of which we evaluate the entire week.

\subsection{Antigen and Antibody Representation}

The data structure that composes each antigen (Ag) data set record and antibody $(\mathrm{Ab})$ detector chromosome is a fixed integer array with binary allele values that define the chromosome's location in the search space. We chose this data structure because it was (conveniently) the same type employed by both REALGO and MISA.

The Abs for network intrusion are generated and trained in the same manner as in anti-virus detectors [13]. However, network 
intrusion Ags are longer and segregated because they utilize the IP packet structure for its template. For this reason, we constrain our ID domain to encode Ags from network packets wrapped in the three most common IP protocols utilized by non-self: TCP, UDP and ICMP. Decimal-value header information from each incoming Ag's packet header field is encoded into its binary equivalent and concatenated to the end of its string. Hence, each Ag's IP $\cup(T C P \vee U D P \vee I C M P)$ header results in a Ag TCP, UDP or ICMP binary DNA chromosome, respectively (Figure 2).

Ab chromosomes are composed of three parts: its DNA (binary), RNA (binary) and seven state attributes (integer) (see Figure 3). Its DNA is generated by negative selection and the only portion of the $\mathrm{Ab}$ to be computed against the Ag. Its RNA is its DNA replica, facilitating the REALGO method of escaping local optima through $R N A$ reverse transcription modeling [5]. If the mutated DNA results in a higher fitness than last time, its DNA is replicated to its personal memory space called RNA. If the fitness is worse, the DNA reverts to its last best fitness RNA to purpose mutation "in a different direction." Finally, there are seven parameters: $\lambda \leftarrow$ name, $\alpha \leftarrow$ number of false detections, $\rho \leftarrow$ (true positive + true negative) fitness score, $\phi \leftarrow$ (false positive + false negative) fitness score, $\eta \leftarrow$ deviation from negative selection-defined affinity threshold (determining volume), $\beta \leftarrow$ broadcasted (yes/no), $\psi \leftarrow$ number of Abs that Pareto-dominate this $\mathrm{Ab}$.

\begin{tabular}{|c|c|c|c|c|c|}
\hline & & \multicolumn{3}{|c|}{1516} & \multirow[t]{2}{*}{31} \\
\hline $\begin{array}{c}\text { 4-bit } \\
\text { version }\end{array}$ & \begin{tabular}{|c|}
$\begin{array}{c}\text { 4-bit header } \\
\text { length }\end{array}$ \\
\end{tabular} & $\begin{array}{l}\text { 8-bit type of service } \\
\text { (TOS) }\end{array}$ & \multicolumn{2}{|r|}{ 16-bit total length (in bytes) } & \\
\hline \multicolumn{3}{|c|}{ 16-bit identification } & \begin{tabular}{|l|l|l|}
0 & $\mathrm{D}$ & $\mathrm{M}$ \\
$\mathrm{F}$ & $\mathrm{F}$ \\
\end{tabular} & 13-bit fragment offset & \\
\hline 8-bit tit & $\begin{array}{l}\text { me to live } \\
\text { (TTL) }\end{array}$ & 8-bit protocol & & 16-bit header checksum & \\
\hline \multicolumn{5}{|c|}{ 32-bit source IP address } & \\
\hline \multicolumn{5}{|c|}{ 32-bit dest ination IP address } & \\
\hline \multicolumn{5}{|c|}{ options (if any) } & \\
\hline \multicolumn{5}{|c|}{ data } & \\
\hline
\end{tabular}

Figure 2. Ag chromosomes are formed through IP (shown), TCP, UDP and ICMP packet header field decimal values.

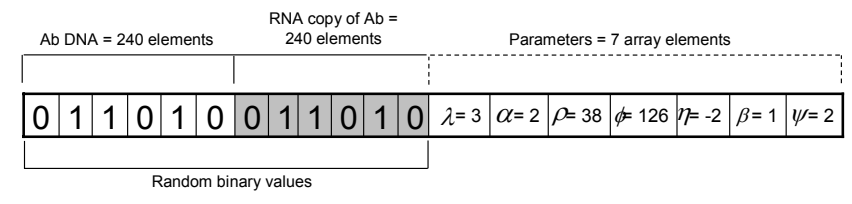

Figure 3. Ab chromosome (DNA $\cup$ RNA $\cup$ parameters).

Because our DNA is composed of a bit string data structure, our affinity measure is Hamming distance (Equation 1). We choose Hamming distance over the r-contiguous bit rule or other measures because we're pattern-matching the entire context of each Ag packet vs. particular contiguous IP fields [11]:

$$
H=\sum_{i=1}^{L} \delta_{i} \text { where } \delta_{i}=\left\{\begin{array}{l}
1 \text { if } A b_{i} \neq A g_{i} \\
0 \text { otherwise }
\end{array}\right.
$$

\subsection{Immune Algorithm}

Integrating REALGO's RNA transcription into the evaluation operator and the MISA framework, including its evaluation, selection and mutation operators, we have the pseudocode for our algorithm we call jREMISA: the Java retrovirus-inspired MISA (Algorithm 1). REALGO and MISA do not employ crossover because of the sufficiency of mutation to move Abs throughout the search space. In addition, crossover can break good building blocks.

\begin{tabular}{|c|c|}
\hline 1 & procedure $\mathrm{jREMISA}$ \\
\hline 2 & begin \\
\hline 3 & repeat \\
\hline 4 & $\begin{array}{c}\text { Randomly generate initial TCP, UDP, ICMP } \\
\text { Populations }\left(\mathrm{P}_{\mathrm{p}}\right)\end{array}$ \\
\hline 5 & Initialize empty secondary Population $\left(\mathrm{P}_{\mathrm{s}}\right)$ \\
\hline 6 & negative_selection $\left(\mathrm{P}_{\mathrm{p}}\right.$, data_set $_{\text {clean }}$, threshold $) / *$ Eval $1 * /$ \\
\hline 7 & until (end of data_set clean $_{\text {) }}$ ) \\
\hline 8 & repeat \\
\hline 9 & fitness function $(\mathrm{ag}) / *$ evaluation_2 $2 * /$ \\
\hline 10 & mutationCauchy $\left(\mathrm{P}_{\mathrm{p}}\right)$ \\
\hline 11 & P_optimality ()$/ *$ evaluation_3*/ \\
\hline 12 & clonalSelection $(0.05)$ \\
\hline 13 & mutationUniform $\left(\mathrm{P}_{\mathrm{s}}\right)$ \\
\hline 14 & $\mathrm{P}_{\mathrm{p}} \leftarrow \mathrm{P}_{\mathrm{s}} / *$ copy best of $\mathrm{P}_{\mathrm{s}}$ as next gen's $\mathrm{P}_{\mathrm{p}} * /$ \\
\hline 15 & if (networking) \\
\hline 16 & broadcast $\left(\mathrm{P}_{\mathrm{s}}\right) / *$ offer nondominateds to all AISs */ \\
\hline 17 & processReceived ()$/ *$ Any captured Abs? $* /$ \\
\hline 18 & endif \\
\hline 19 & until (end of data_set $t_{\text {attack }}$ ) \\
\hline 20 & end \\
\hline
\end{tabular}

Algorithm 2. jREMISA AIS-inspired MOEA.

Algorithm 2, lines 3-7, which equates to Algorithm 1, lines 3-5, consist of negative selection where Abs are randomly generated and evaluated against every Ag of a self-only day of traffic, given a predefined affinity threshold percentage. If an $\mathrm{Ab}$ reacts to self, it is discarded without replacement. In doing this, we are assured that the remaining population at algorithm termination does not react to a single packet of the day's traffic. Post-negative selection consists of Algorithm 2, lines 8-19, which equates to Algorithm 1, lines 6-12. These lines are summed up in Figure 4 where each Ag entering the evaluation window represents a new generation. We partition our primary population $\left(\right.$ pop $\left._{\mathrm{p}}\right)$ by IP protocol for two reasons: efficiency is increased by evaluating the $\mathrm{Ag}$ only against a subset of pop $\mathrm{p}_{\mathrm{p}}$ and pattern-matching becomes more relevant. E.g., TCP, UDP and ICMP don't all share the same chromosomal structure, as their IP fields differ; hence, it doesn't make sense to compare a TCP Ab to a non-TCP Ag. 


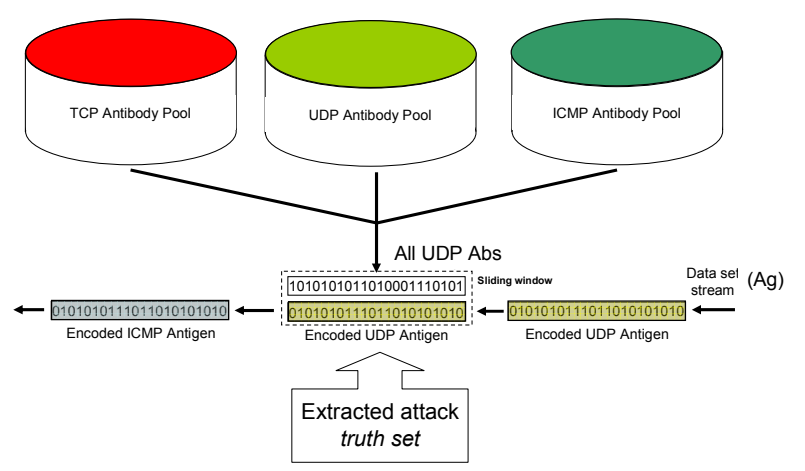

Figure 4. Transient (data set) Ags evaluated against its IP protocol-matching Ab.

When the Ag enters the window, the following occurs (per Algorithm 2):

- $\quad$ FITNESS FUNCTION (line 9): Hamming distance $H$ is computed between the $\mathrm{Ab}$ and $\mathrm{Ag}$ DNA signature. Combined with the affinity threshold and truth set informing whether this Ag is self or non-self, one of four outcomes results:

$\circ \quad$ True negative $(\mathrm{Ag} \leftarrow \mathrm{Ab} \leftarrow$ self $)$ : penalize first objective $\left(\mathrm{obj}_{1}\right)$ fitness $+=H$, copy DNA to RNA, reward second objective $\left(\mathrm{obj}_{2}\right)+=1 \%$;

$\circ \quad$ True positive $(\mathrm{Ag} \leftarrow \mathrm{Ab} \leftarrow$ non-self $):$ penalize $\mathrm{obj}_{1}$ $+=\left(\right.$ Ag length $\left._{\text {lo }}-H\right)$, copy DNA to RNA, reward $\mathrm{obj}_{2}$ $+=1 \%$;

$\circ \quad$ False negative $(\mathrm{Ag} \leftarrow$ self, $\quad \mathrm{Ab} \leftarrow$ non-self $)$ : falseDetections ++ , revert DNA to RNA, penalize $\mathrm{obj}_{2}-=1 \%$;

$\circ \quad$ False positive $(\mathrm{Ag} \leftarrow$ non-self, $\quad \mathrm{Ab} \leftarrow$ self $):$ falseDetections ++ , revert DNA to RNA, penalize $\mathrm{obj}_{2}-=1 \%$.

Both true negative and positive outcomes are penalized because, if true negative, $H$ should ideally equal zero. If not, $\mathrm{obj}_{1}$ 's value is increased by the number of complimentary alleles $(H)$. If true positive, then $H$ should ideally equal the length of the Ag. If not, obja's value is increased by the number of non-complementary alleles;

- CAUCHY MUTATION (line 10): REALGO suggests Cauchy mutation to move Abs around the landscape. We perform it upon all penalized $\mathrm{Ab}$ alleles;

- $\quad P^{*}$-TEST (line 11): all Abs undergo a Pareto-optimality test to determine the number of Abs each is dominated by. Quicksort then ascending-sorts them;

- MISA's CLONAL SELECTION PRINCIPLE (lines 12-14): elitist selection copies the top 5\% of nondominated Abs from their pop $_{\mathrm{p}}$ to their respective secondary (or external) population $\left(\mathrm{pop}_{\mathrm{s}}\right.$ ) meant to contain only nondominated Abs. Copied Abs are cloned to $600 \%$ the pops size. All copied and cloned Abs are then mutated in $n$-random allele positions, where $n \leftarrow$ is the number of objectives (two) plus their Pareto-dominance value. Following, any pop $\mathrm{p}_{\mathrm{p}} \mathrm{Abs}$ lost to reaching the max number of false detections is replaced by the fittest Abs from the pop $p_{s}$ in order to return the pop $p_{p}$ to its original size. Finally, the pop $_{\mathrm{s}}$ is culled for only nondominated Abs;
- AD-HOC NETWORKING (optional): if enabled, newly discovered nondominated Abs copied to the pop s $_{\mathrm{s}}$ are broadcast to the subnet where listening jREMISAs capture and add to their pops only if it dominates their entire pops (see Figure 5).

This process defines one generation and recurs for the number of data set packets.

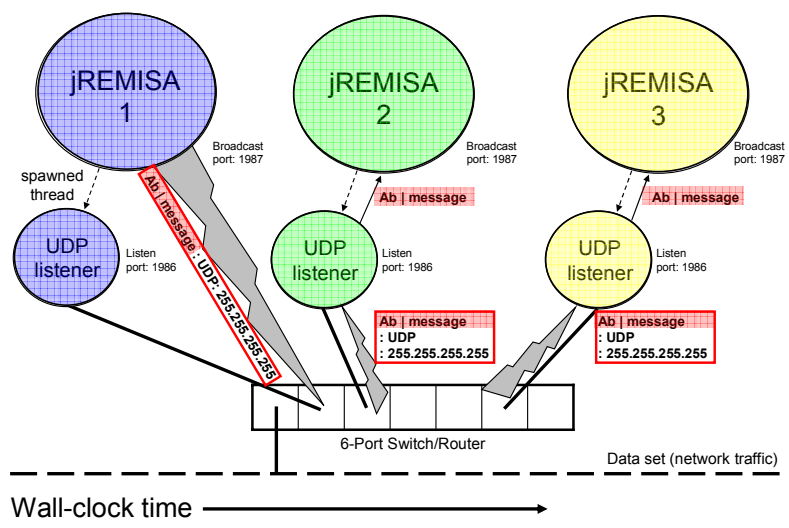

Figure 5. jREMISA distributed communication architecture.

The software engineering of jREMISA in design and implementation used various paradigms; Model-View-Controller, UML Class Diagram, and Design Patterns[4]. jREMISA was developed in the Eclipse ${ }^{2}$ open-source Integrated Development Environment (IDE).

\section{EXPERIMENTATION AND ANALYSIS}

Based upon the objectives of Section 2, the experiments are divided into three parts:

1. validation of the C-to-Java migration of REALGO and MISA;

2. jREMISA effectiveness measurement against the LL data set in 13 scenarios: 10 standalone, involving all days of week two, and three distributed island model executions in a two-, three- and four-jREMISA configuration;

3. statistically comparing jREMISA against other algorithms applied to the same data set.

In the distributed configuration, four computers with Windows XP Professional 2002, Service Pack 2 were used

- $\quad$ "PC1" $\leftarrow$ Dell Inspiron 710m laptop, 2 GHz Pentium-M, 2 GB of RAM

- "PC2" $\leftarrow$ Dell XPS laptop, 3.4 GHz Pentium-4 HyperThreading, 1 GB RAM;

- "PC3" $\leftarrow$ Dell Precision laptop, $1.8 \mathrm{GHz}$ Pentium-4, 512 MB RAM;

- $\quad$ "PC4" $\leftarrow$ Dell Optiplex GX270 tower, 2.6 GHz Pentium-4, 512 MB RAM.

The C-to-Java migration experiment is straightforward, yet we are concerned about the impact of a different programming language and random number generator on the output. Figure 6 compares the output between REALGO and jREALGO, employing REALGO's Yao and Liu's test function [6]where the optimal value is -12569.5 . Figure 7 compares the output between MISA

\footnotetext{
${ }^{2}$ The Eclipse project, www.eclipse.org.
} 
and jMISA, employing MISA's Kita-proposed two-variable test function [5].

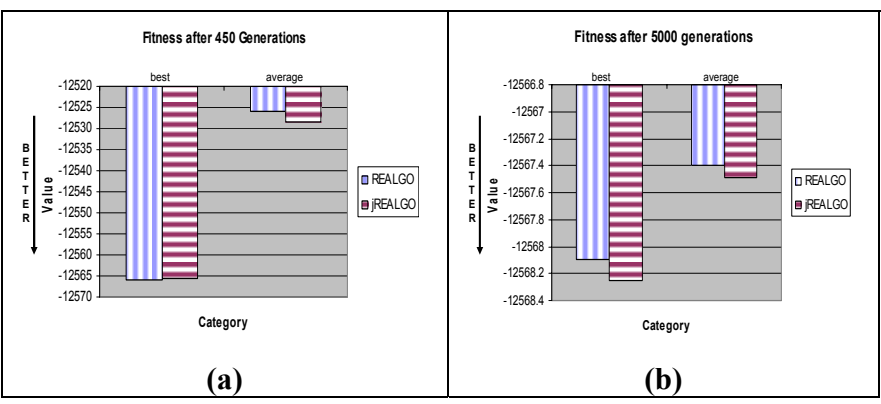

Figure 6. REALGO vs. jREALGO: (a) after 450 generations; (b) after 5000 generations.

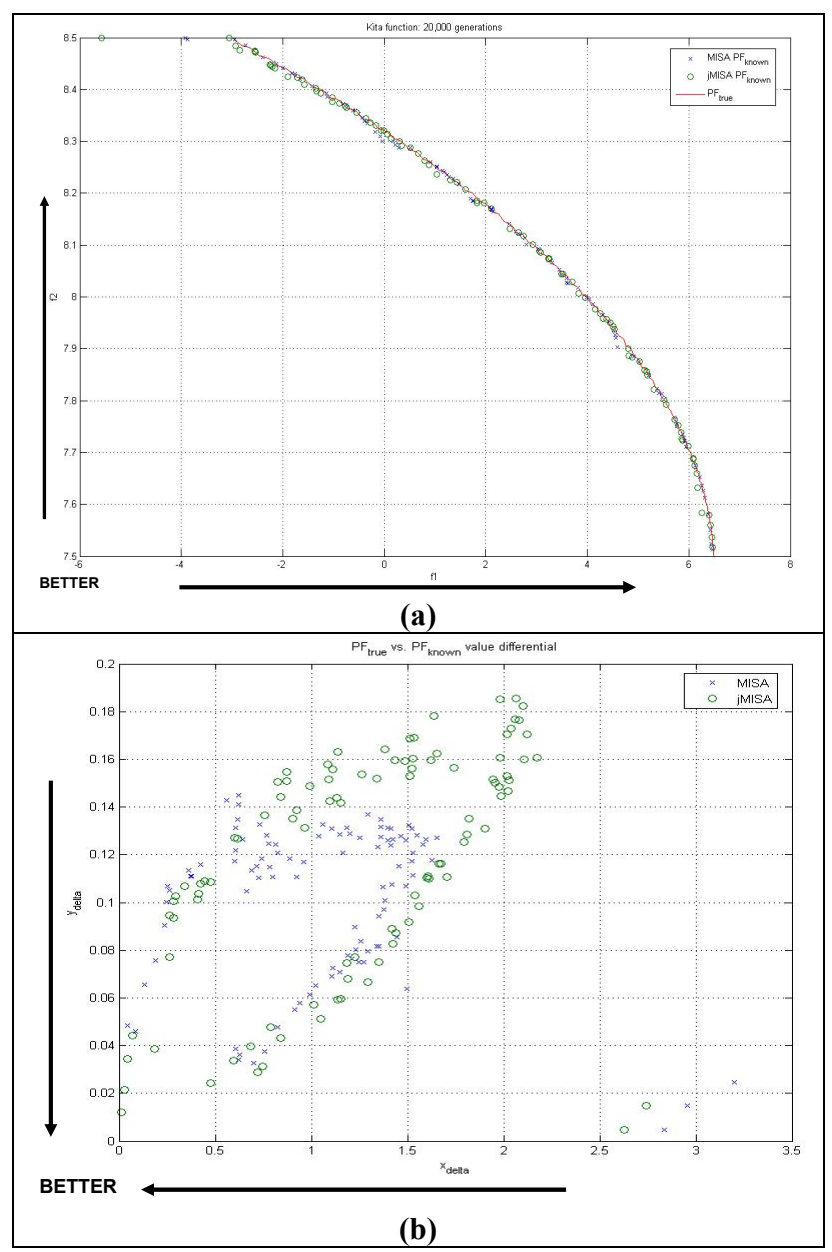

Figure 7. (a) MISA and jMISA $P F$ vs. MISA's true $P F^{*}$; (b) Euclidian distance measure of MISA and jMISA points from MISA's true $P F^{*}$.

While Figure 7(a) shows MISA and jMISA's known $P F$ to be almost identical, Figure 7(b)'s Euclidian distance measurement of each point shows that while MISA has the preponderance of points closer to true $P F^{*}$, jMISA possesses the few points closest to true $P F^{*}$. Therefore, based on the comparison of REALGO to
jREALGO and MISA to jMISA, we conclude this experiment validated.

Mapping the LL truth set of labeled-attacks to the Ethereal ${ }^{3}$ analyzed five days of labeled attacks, 16 context-based attacks are extracted (Figure 8).

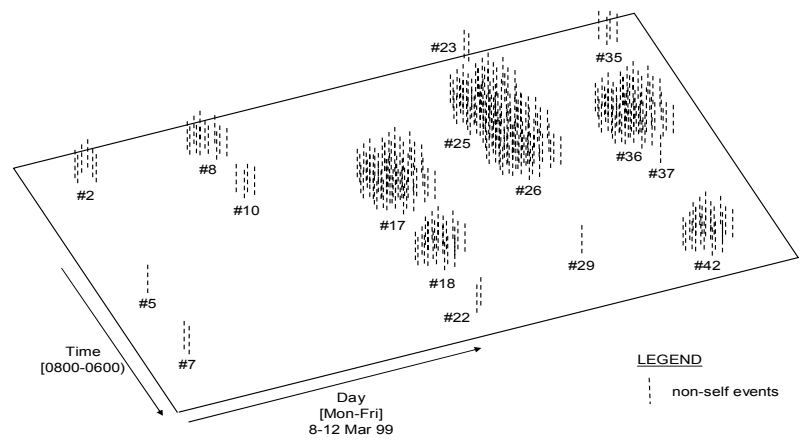

Figure 8. LL 1999 week-two insider data set landscape with labeled attacks.

In comparing our results to Williams' Warthog [14], the surviving

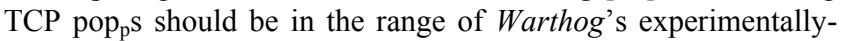
employed Abs: between 32 and 2048. Table 1 shows that this Ab range requires the affinity threshold between $37-42 \%$. In Table 2, scenarios 1-6 show our Ab effectiveness based on each threshold. Since scenario 4 has the combined highest classification and lowest false detection rate, we use that $39 \%$ threshold to compare it to the remaining days of the week (scenarios 7-10). In Table 3, we employ this same threshold to the distributed tests, differing only by the number of partitions in the data set decomposition.

Figure 10(a) shows the scenario-four TCP pop . This PF* is provided for all three populations for each MOEA execution. Its intent is to show the tradeoff between each Abs fitness score and affinity threshold deviation. For all five days of the week, all three pop $P F^{*}$ had Abs in the $+(4-5) \%$ range $73 \%$ of the time. In addition, Abs were concentrated in this same range $87 \%$ of the time. Figure 10(b) shows an attack graph where each Ab plots its classification declaration for each attack. The $\mathrm{x}$-axis indicates the outcome while the $y$-axis represents the packet number, mapping the attack. Here, there are no points on the false positive side for two attacks, indicating a $0 \%$ false positive rate for LL attack \#26 and \#29. While not shown, jREMISA also optimally discovered LL attacks \#7 and \#22.

Figure 11 depicts a snapshot of PC1 in scenario 11 where PC1 has broadcast $\mathrm{Abs}$ and decided to reject an incoming $\mathrm{Ab}$ received from PC2. Figure 15 shows how our algorithm does better than Warthog with regard to the false detection rate as the number of Abs are increased for the week-two Thursday data set. With Warthog, Williams declares that as the number of Abs increases, so does the false positive rate. However, our experiments show that as we increased our Abs, the false positive rate decreased. Therefore, we have shown how our algorithm is more effective with respect to the same benchmark data set.

\footnotetext{
${ }^{3}$ Ethereal: open-source network protocol analyzer, www.ethereal.com.
} 
Figure 12 graphically depicts Table 2 with classification and false detection ratios having $5 \%$ variance due to the multiple trials run of the same scenario to maximize statistical accuracy. Figure 13 and Figure 14 summarize the results of the distributed phase of our experiments. In Figure 13, a graphical depiction of Table 3, we discover that the sharing of nondominated Abs among jREMISAs did not conclusively show a synergistic increase in effectiveness when compared to the standalone classification ratio, although the two-PC configuration fared better. Figure 14 shows the increase in efficiency as more jREMISAs participated. Upon the completion of either phase-negative selection or MOEA - jREMISA saves the resulting

population, Pareto Front and attack graph values to a formatted XML file (Figure 9)

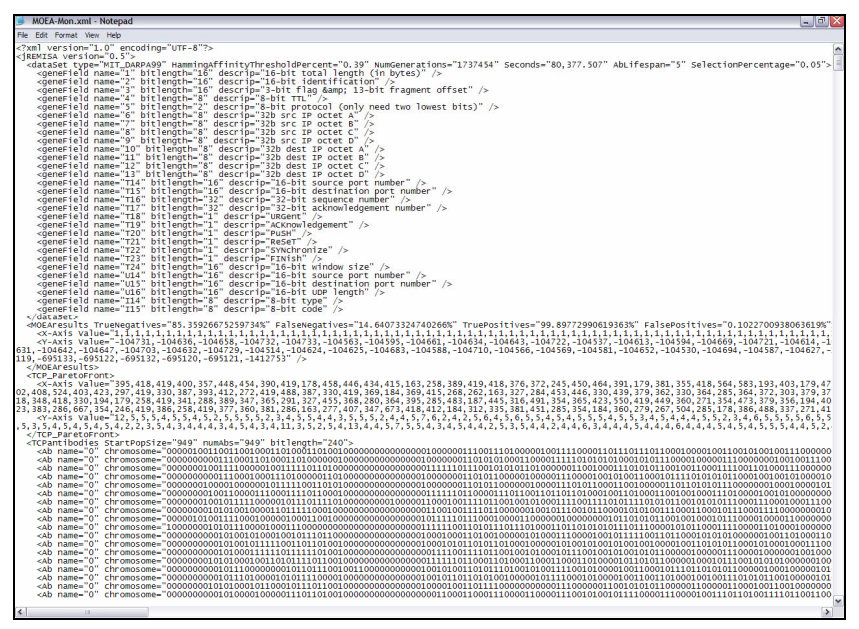

Figure 9: Example post-MOEA XML output file

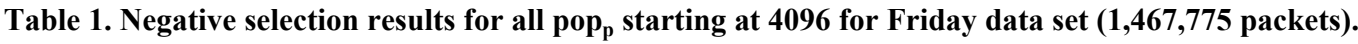

\begin{tabular}{|l|l|l|l|l|l|l|l|}
\hline Affinity (\%) & Runtime(mins) & End TCP & survived & End UDP & survived & End ICMP & survived \\
\hline 37 & 186.65 & 2663 & $65.015 \%$ & 3737 & $91.235 \%$ & 3707 & $90.503 \%$ \\
\hline 38 & 124.20 & 1563 & $38.159 \%$ & 3372 & $82.324 \%$ & 3513 & $85.767 \%$ \\
\hline 39 & 89.17 & 935 & $22.827 \%$ & 2890 & $70.557 \%$ & 3290 & $80.322 \%$ \\
\hline 40 & 45.27 & 357 & $8.716 \%$ & 2275 & $55.542 \%$ & 2700 & $65.918 \%$ \\
\hline 41 & 26.43 & 126 & $3.076 \%$ & 2000 & $48.828 \%$ & 2344 & $57.227 \%$ \\
\hline 42 & 16.28 & 34 & $0.830 \%$ & 1431 & $34.937 \%$ & 1997 & $48.755 \%$ \\
\hline
\end{tabular}

Table 2. MOEA run summary: standalone jREMISA.

\begin{tabular}{|c|c|c|c|c|c|c|c|c|c|c|c|}
\hline \multirow{3}{*}{$\begin{array}{l}\text { Scen- } \\
\text { ario }\end{array}$} & \multirow[b]{2}{*}{ Day } & \multirow[b]{2}{*}{$\begin{array}{l}\text { Gener- } \\
\text { ations }\end{array}$} & \multirow[b]{2}{*}{$\begin{array}{l}\text { Affinity } \\
\text { Threshold }\end{array}$} & \multirow[b]{2}{*}{$\begin{array}{l}\text { TCP } \\
\text { Pop }\end{array}$} & \multirow[b]{2}{*}{$\begin{array}{l}\text { UDP } \\
\text { Pop }\end{array}$} & \multirow[b]{2}{*}{$\begin{array}{l}\text { ICMP } \\
\text { Pop }\end{array}$} & \multirow[b]{2}{*}{ Runtime } & \multicolumn{2}{|c|}{ Self Events } & \multicolumn{2}{|c|}{ Non-self Events } \\
\hline & & & & & & & & $\begin{array}{l}\text { True } \\
\text { Neg\% }\end{array}$ & $\begin{array}{l}\text { False } \\
\text { Neg\% }\end{array}$ & $\begin{array}{l}\text { True } \\
\text { Pos } \%\end{array}$ & $\begin{array}{l}\text { False } \\
\text { Pos } \%\end{array}$ \\
\hline & Thurs & 1547710 & $42 \%$ & 37 & 86 & 248 & $39.12 \mathrm{~m}$ & 53.78 & 46.22 & 62.6 & 37.4 \\
\hline 2 & " & " & $41 \%$ & 106 & 116 & 284 & $52.48 \mathrm{~m}$ & 67.44 & 32.56 & 68.33 & 31.67 \\
\hline 3 & “ & “ & $40 \%$ & 315 & 146 & 341 & $3.61 \mathrm{hrs}$ & 76.10 & 23.90 & 76.92 & 23.08 \\
\hline 4 & " & “ & $39 \%$ & 966 & 361 & 810 & $18.21 \mathrm{hrs}$ & 85.45 & 14.55 & 97.66 & 2.34 \\
\hline 5 & “ & “ & $38 \%$ & 1580 & 423 & 881 & 2.36 day & 86.48 & 13.52 & 92.51 & 7.49 \\
\hline 6 & "“ & “" & $37 \%$ & 2564 & 462 & 927 & 5.83 day & 82.52 & 17.48 & 99.71 & 0.29 \\
\hline 7 & Mon & 1737455 & $39 \%$ & 969 & 349 & 846 & $20.02 \mathrm{hr}$ & 85.36 & 14.64 & 99.90 & 0.10 \\
\hline 8 & Tues & 1571748 & “" & 922 & 362 & 882 & $18.86 \mathrm{hr}$ & 84.61 & 15.39 & 97.35 & 2.65 \\
\hline 9 & Wed & 995235 & " & 920 & 333 & 798 & $11.69 \mathrm{hr}$ & 83.37 & 16.63 & 98.26 & 1.74 \\
\hline 10 & Fri & 1347393 & " & 964 & 376 & 829 & $13.43 \mathrm{hr}$ & 83.59 & 16.41 & 96.57 & 3.43 \\
\hline
\end{tabular}

Table 3. MOEA run summary: distributed jREMISAs against Thursday data set.

\begin{tabular}{|c|c|c|c|c|c|c|c|c|c|}
\hline & & & & & & \multicolumn{2}{|c|}{ Self Events } & \multicolumn{2}{|c|}{ Non-self Events } \\
\hline $\begin{array}{l}\text { jREMISA } \\
\text { ID }\end{array}$ & $\begin{array}{l}\text { Packet range } \\
\text { (1547709 total) }\end{array}$ & $\begin{array}{l}\text { TCP } \\
\text { Pop }\end{array}$ & $\begin{array}{l}\text { UDP } \\
\text { Pop }\end{array}$ & $\begin{array}{l}\text { ICMP } \\
\text { Pop }\end{array}$ & Runtime & $\begin{array}{l}\text { True } \\
\text { Neg} \%\end{array}$ & $\begin{array}{l}\text { False } \\
\mathrm{Neg} \%\end{array}$ & $\begin{array}{l}\text { True } \\
\text { Pos } \%\end{array}$ & $\begin{array}{l}\text { False } \\
\text { Pos } \%\end{array}$ \\
\hline \multicolumn{10}{|c|}{ Scenario $11: 2$ jREMISAs, $39 \%$ affinity threshold, Thursday attack data set } \\
\hline PC1 & $1-773854$ & 966 & 361 & 810 & $9.44 \mathrm{hrs}$ & \multirow{2}{*}{86.21} & \multirow{2}{*}{13.79} & \multirow{2}{*}{98.10} & \multirow{2}{*}{1.90} \\
\hline $\mathrm{PC} 2$ & $773855-1547709$ & 936 & 344 & 854 & $9.63 \mathrm{hrs}$ & & & & \\
\hline \multicolumn{10}{|c|}{ Scenario $12: 3$ jREMISAs, $39 \%$ affinity threshold, Thursday attack data set } \\
\hline PC1 & $1-515903$ & 966 & 361 & 810 & $5.09 \mathrm{hrs}$ & \multirow{3}{*}{84.31} & \multirow{3}{*}{15.69} & \multirow{3}{*}{97.94} & \multirow{3}{*}{2.06} \\
\hline $\mathrm{PC} 2$ & $515904-1031807$ & 936 & 344 & 854 & $6.35 \mathrm{hrs}$ & & & & \\
\hline PC3 & $1031808-1547709$ & 951 & 357 & 826 & $6.86 \mathrm{hrs}$ & & & & \\
\hline \multicolumn{10}{|c|}{ Scenario $13: 4$ jREMISAs, $39 \%$ affinity threshold, Thursday attack data set } \\
\hline $\mathrm{PC} 1$ & $1-386927$ & 966 & 361 & 810 & $4.33 \mathrm{hrs}$ & \multirow{4}{*}{84.94} & \multirow{4}{*}{15.06} & \multirow{4}{*}{98.55} & \multirow{4}{*}{1.45} \\
\hline $\mathrm{PC} 2$ & $386928-773854$ & 936 & 344 & 854 & $4.63 \mathrm{hrs}$ & & & & \\
\hline PC3 & $773855-1160781$ & 951 & 357 & 826 & $4.86 \mathrm{hrs}$ & & & & \\
\hline PC4 & $1160782-1547709$ & 954 & 360 & 822 & $5.09 \mathrm{hrs}$ & & & & \\
\hline
\end{tabular}




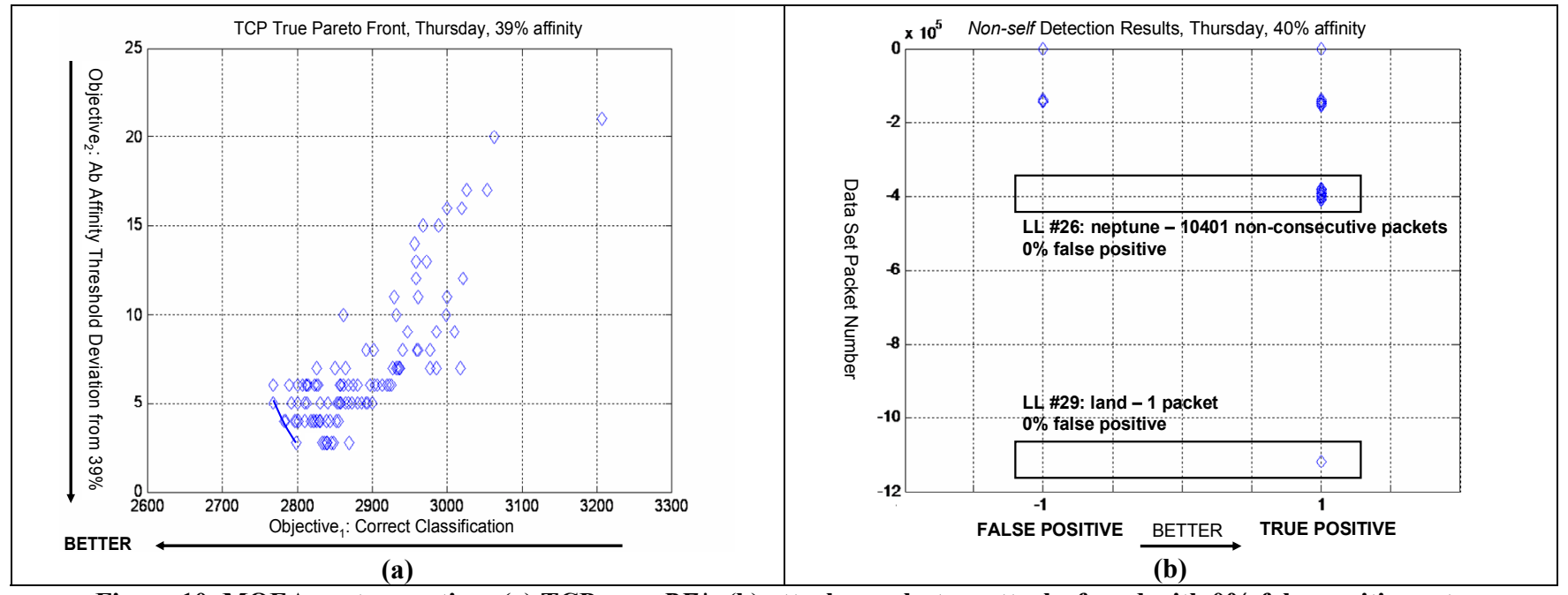

Figure 10. MOEA post-execution: (a) TCP pops $P F^{*}$; (b) attack graph: two attacks found with $0 \%$ false positive rate.

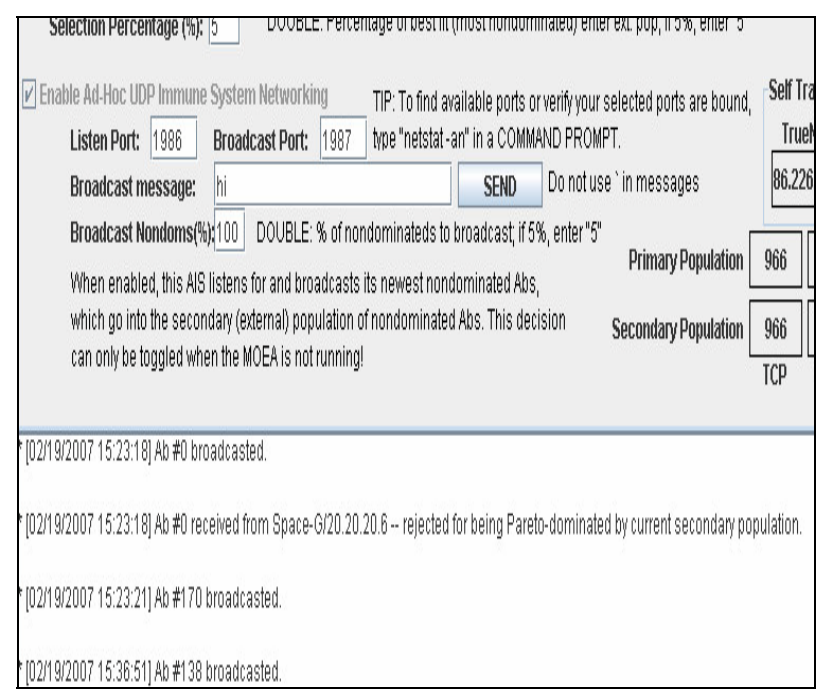

Figure 11. Snapshot of jREMISA distributed communication.

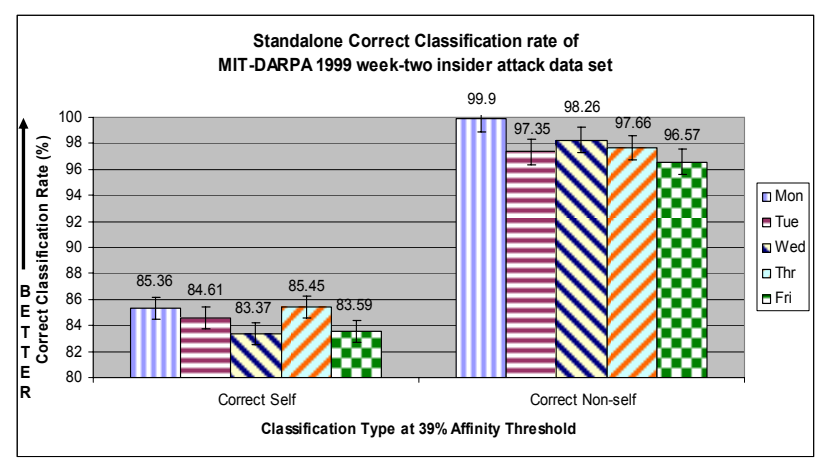

Figure 12. jREMISA standalone effectiveness against each day of the week-two insider attack data set.

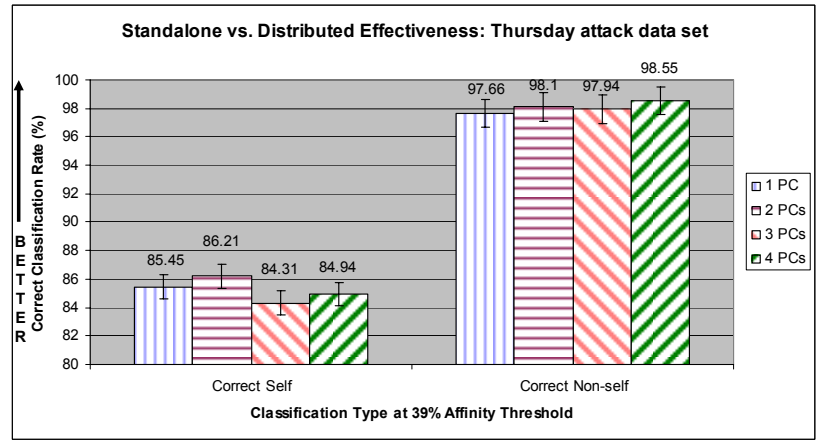

Figure 13. Standalone vs. distributed effectiveness against the week-two Thursday insider attack data set.

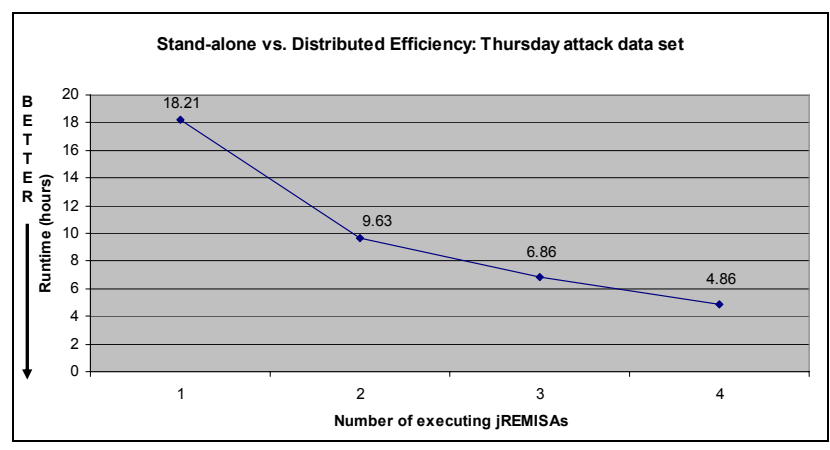

Figure 14. Data decomposition-based distributed execution: efficiency vs. number of executing jREMISAs. 


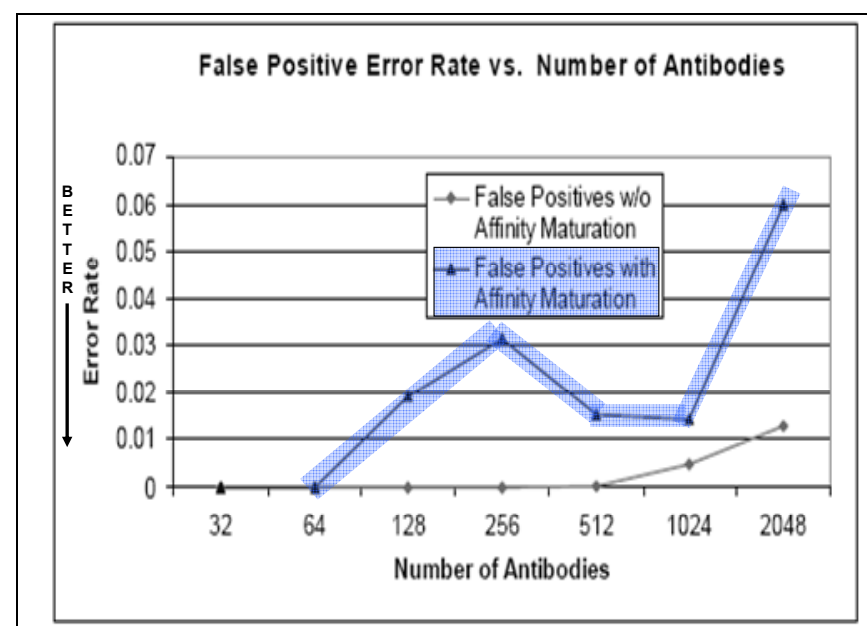

(a)

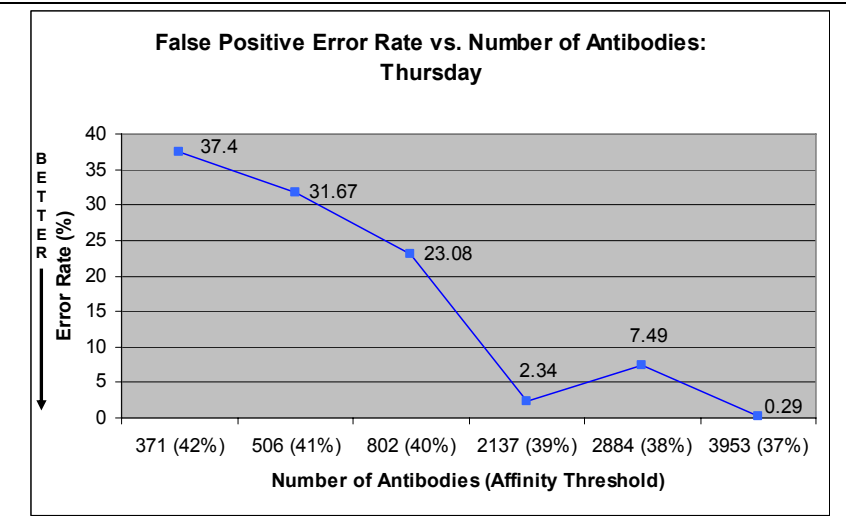

(b)

Figure 15. false positive error rate trend: (a) Warthog [15] vs. (b) jREMISA.

\section{CONCLUSION}

The hypothesis was tested against four measurable goals which were all validated based on our experimentation. REALGO and MISA were successfully migrated to Java (and integrated via software engineering principles into jREMISA). Our algorithm achieved an average $83.37-85.45 \%$ self classification and $96.57-$ $99.90 \%$ non-self classification rate for a $39 \%$ affinity threshold. We observed a patterned $\mathrm{Ab}$ hypervolume between $0-5 \%$ above this threshold (making their hypervolume 39-44\%) and $\mathrm{Ab}$ broadcasting and receipt. In addition, we validated our algorithm as performing better in at least one way over another against the same data set. As a bonus, jREMISA detected four attacks, ranging from one to 10401 non-consecutive packets, with a $0 \%$ false positive rate. From this, we declare this IDS as possibly the first validated AIS-inspired MOEA applied to the ID problem domain.

Use of software engineering design paradigms provides an easily extendable IDS package. Future jREMISA advances include com pleting the KDD Cup 99 data set facilitation, evolving the $\mathrm{Ab}$ hypershape and exploring advanced negative selection methodologies.

\section{REFERENCES}

[1] Symantec Internet Security Threat Report, Trends for January 1, 2004 - June 30, 2004, Volume VI, September, 2004, eval.veritas.com/mktginfo/enterprise/white_papers/entwhitepaper_symantec_internet_security_threat_report_vi.pdf

[2] Michalewicz, Z., Fogel, D., How to Solve It: Modern Heuristics, Second Edition, Springer 2004.

[3] J.. Dréo, A. Pétrowski, P. Siarry, and E. Taillard, Metaheuristics for Hard Optimization: Methods and Case Studies, Springer-Verlag, Berlin, Germany, 2006.

[4] Haag, C.R., An Artificial Immune System-inspired Multiobjective Evolutionary Algorithm with Application to the Detection of Distributed Computer Network Intrusions, M.S. Thesis, Graduate School of Engineering and Management, Air Force Institute of Technology, WPAFB, Dayton, OH, March, 2007

[5] Coello, C., Cortés, N., Solving Multiobjective Optimization Problems Using an Artificial Immune System, Genetic Programming and Evolvable Machines, Vol. 6, pp.163-190, 2005.

[6] Edge, K., Lamont, G., Raines, R., A Retrovirus Inspired Algorithm for Virus Detection \& Optimization, GECCO '06, July 8-12, 2006.

[7] McGee, P., Building Better Antibody Therapeutics, Drug Discovery \& Development, www.dddmag.com/ShowPR.aspx?PUBCODE=090\&ACCT= $1600000100 \&$ ISSUE $=0701 \&$ RELTYPE $=$ DEV \&PRODCOD $\mathrm{E}=00000000 \& \mathrm{PRODLETT}=\mathrm{AG} \&$ CommonCount $=0$.

[8] Middlemiss, M., Positive and Negative Selection in a Multilayer Artificial Immune System, Information Science Discussion Paper Series, No. 2006/03, University of Otago, January, 2006.

[9] Coello, C., Van Veldhuizen, D., Lamont, G.B., Evolutionary Algorithms for Solving Multi-Objective Problems, Springer 2002.

[10] De Castro, L.N., Timmis, J., Artificial Immune Systems: A New Computational Intelligence Approach, Springer 2002.

[11] MIT Lincoln Laboratory-DARPA Intrusion Detection Evaluation, www.1l.mit.edu/IST/ideval/.

[12] Mahoney, M., Chan, P., An Analysis of the 1999 DARPA/Lincoln Laboratory Evaluation Data for Network Anomaly Detection, Technical Report CS-2003-02, Computer Science Department, Florida Institute of Technology, 2003.

[13] Harmer, P., Williams, P., Gunsch, G., Lamont, G.B., An Artificial Immune System Architecture for Computer Security Applications, IEEE Transactions on Evolutionary Computation, Vol. 6, No. 3, June 2002.

[14] Williams, P., WARTHOG: Towards a Computer Immune System for Detecting "Low and Slow" Information System Attacks, AFIT Master's Thesis, March, 2001 